\title{
Estudo do potencial de um sintetizador de voz no processo educativo de uma criança com ataxia
}

\author{
Ana Teresa Oliveira* \\ António JoséOsório** \\ Anabela CruzSantos***
}

\section{Resumo}

Este estudo de caso analisa o uso das Tecnologias de Informação e Comunicação (TIC) no apoio a uma criança com Ataxia, a Bia (nome fictício) incluída numa Unidade de Apoio Especializado para a educação de alunos com Multideficiência (UAEM), numa escola pública de Braga, Região Norte de Portugal. A Bia apresentava incapacidade total ao nível da oralidade e graves dificuldades na coordenação da motricidade fina. Além de uma contextualização da relação entre as necessidades educativas especiais (NEE) e as TIC, através da sua interligação, aborda-se e descreve-se a intervenção ao nível das TIC e a sua integração no currículo e na aprendizagem e em seguida relata-se este processo de integração, que teve a finalidade de lhe proporcionar produtos de apoio que assegurassem os necessários meios para promover e aumentar a sua comunicação.

Palavras-chave: Inclusão; Necessidades Educativas Especiais (NEE); Ataxia; Tecnologias de Informação e Comunicação (TIC); Sintetizador de Voz.

\footnotetext{
* Mestre em Estudos da Criança, Área de Especialização em Tecnologias de Informação e Comunicação, Universidade do Minho, Braga, Portugal.

** Professor Auxiliar com Agregação, Phd, Departamento de Estudos Curriculares e Tecnologia Educativa do Instituto de Educação da Universidade do Minho, Braga, Portugal.

*** Professora Auxiliar, Phd do Departamento de Psicologia da Educação e Educação Especial, Universidade do Minho, Braga, Portugal.
} 


\title{
Study of the potential of a voice synthesizer in the educational process of a child with ataxia
}

\begin{abstract}
This case study examines the use of Information and Communication Technologies (ICT) in supporting Bia (a fictitious name), a child with Ataxia, included in a Support Unit for the education of students with multiple disabilities of a school in Braga, Northern Portugal. Bia presented total incapacity in speech and severe difficulties in fine motor coordination. Thus, this study presents the relationship between special educational needs and ICT through their interconnection. In addition, this research addresses and describes the intervention in terms of ICT and its integration into the curriculum and Bias's learning process. The integration process is reported in order to provide supporting products for augmentative and alternative communication.
\end{abstract}

Keywords: Inclusion; Special Educational Needs; Ataxia; Information and Communication Technologies (ICT); Voice Synthesizer.

\section{Introdução}

Este texto apresenta o estudo de caso da Bia (nome fictício), uma criança com Ataxia, e do seu processo educativo. A Bia encontrava-se inserida numa escola pública do distrito de Braga, Região Norte de Portugal, numa Unidade de Apoio Especializado para a educação de alunos com Multideficiência (UAEM) - regulamentadas pelo Decreto- Lei 3/2008 (7 de janeiro), art.. ${ }^{26 .}$, inseridas em agrupamentos de escolas e concentrando meios humanos e materiais que possam oferecer uma resposta educativa de qualidade. A Bia apresentava dificuldades significativas e permanentes ao nível da comunicação verbal. No entanto, expressava-se utilizando gestos, expressões faciais e corporais e iniciava turnos comunicativos, com bastante facilidade, com os diferentes interlocutores. A Bia apresentava um estilo de relacionamento interpessoal sociável, mas deparava-se, sempre, com a dificuldade dos outros em compreenderem a sua mensagem e, consequentemente, com ela manter e concluir os turnos comunicativos. Refletindo sobre as interações iniciais com a Bia e analisando o seu percurso educativo, pareceu necessário repensar as práticas ao nível da intervenção junto da aluna, designadamente através do recurso a produtos de apoio adequados à sua problemática.

Assim, este texto inclui uma parte inicial que fundamenta a exploração das TIC na educação de crianças com NEE, após o que descreve o trabalho realizado com a Bia, durante um ano letivo, com vista a proporcionar-lhe meios de comunicação aumentativa e alternativa (CAA). Tendo sido adotada uma metodologia de estudo de caso, a parte final do texto procura sintetizar as principais aprendizagens realizadas, as quais podem ser de relevante utilidade para a investigação e para os profissionais com interesse nestas temáticas. 


\section{Necessidades Educativas Especiais e Tecnologias de Informação e Comunicação}

Por necessidades educativas especiais entende-se o conjunto de alunos que, por apresentarem determinadas condições específicas, podem necessitar de apoio de serviços de educação especial durante todo ou parte do seu percurso académico, de forma a desenvolverem-se ao nível escolar, pessoal e socioemocional (CORREIA, 2008). Desta forma, este conceito aplica-se a crianças e adolescentes com problemas sensoriais, físicos e de saúde, intelectuais e emocionais que necessitem usufruir dos serviços de educação especial que CORREIA (2008) define como o conjunto de recursos que prestam serviços de apoio especializados ao nível académico, terapéutico, social, clínico/médico ou psicológico. Estes tipos de serviços devem ser implementados, quando possível, na sala de aula, tendo como finalidade a prevenção, a redução ou a eliminação dos problemas apresentados pela criança, e /ou a alteração dos seus ambientes de aprendizagem de forma a receber uma educação, por direito, de acordo com as suas necessidades e capacidades (CORREIA, 2008), sendo a escola a responsável pela resposta eficaz e inclusão destes alunos. A inclusão implica uma mudança de paradigma, uma atitude de crença e um sentido de pertença; uma intensa e efetiva mudança nos valores e práticas educativas. Como sublinha RODRIGUES (1997) "a inclusão não é uma cosmética educativa que pode cobrir a escola tradicional (...) constitui um desafio radical à escola, tal como ela se encontra organizada" (p. 12). A participação é vista como um direito básico que assiste a todos e não um privilégio, logo, não deve estar dependente de qualquer gênero de pré-requisito por parte das crianças, seja de carácter acadêmico ou até social, o que conduz a uma completa remodelação ou reestruturação das escolas regulares, com o objetivo de receber e responder, eficazmente, às diferentes necessidades de todas as crianças. Tal como defende CORREIA (2008, p.22), "A inclusão exige, assim a reestruturação da escola e do currículo de forma a permitir aos alunos com NEE uma aprendizagem em conjunto".

Entretanto, o desenvolvimento vertiginoso das TIC mudou o mundo, e tornou-se urgente redefinir as prioridades educativas pois, como refere ILHARCO (2004, p. 48-49), a "tecnologia não é apenas uma nova forma de fazer as mesmas coisas, mas, de facto, uma coisa nova, uma nova forma de estar e de ser, por isso, um novo mundo". Tornou-se imperioso repensar os modelos pedagógicos tradicionais, pois sabemos agora que quantos mais sentidos entrarem na aprendizagem, mais esta se torna eficaz. A utilização do computador, do vídeo e da Internet abriu ao processo de ensino-aprendizagem muitas possibilidades ainda por explorar. Numa sala de aula, com diferentes equipamentos e aplicações, o professor pode criar interatividade, estimulando, nos alunos, a capacidade de iniciativa, de investigação e desenvolvendo o pensamento crítico, bem como rentabilizar e potenciar as capacidades comunicacionais entre ambos.

A versatilidade das TIC é enorme e põe à disposição dos alunos e dos professores um manancial inesgotável de informação com a capacidade de oferecerem novas formas de armazenamento e consulta da informação, que vão para além da linguagem verbal e podem conter som, gráficos, vídeo, animação ou outros media. PAPERT (1994) alertava para que a "melhor aprendizagem ocorre quando o aprendiz 
assume o comando de seu próprio desenvolvimento em atividades que sejam significativas e que lhe despertem o prazer" (p. 29).

As vantagens que as tecnologias trazem à educação, em geral, são também em vários aspetos, comuns à educação especial, e são, em muitos os casos, um meio de acesso ao currículo, convertendo-se num recurso importante para novas aprendizagens e facilitadoras da Inclusão, constituindo um meio fundamental para alunos com problemas ao nível sensorial, motor ou intelectual atingirem níveis de desempenho superiores (RODRIGUES, MORATO, MARTINS, \& CLARA 1991)

\section{Descrição do uso do computador e do sintetizador de voz no processo educativo da Bia}

Nesta parte do texto, procede-se à apresentação do caso estudado, tendo como preocupação central uma descrição pormenorizada das características e do contexto da Bia e do trabalho com ela realizado no seu contexto educativo, no período de maio de 2009 a junho de 2010, de forma a introduzir sistemas de CAA.

A informação necessária para a apresentação deste caso, que na sua descrição seguirá uma ordenação cronológica crescente, foi recolhida através de registos de observação de atividades, acontecimentos e comportamentos em vários contextos relacionados com o processo educativo da Bia, na sequência da atividade profissional da primeira autora, como professora de educação especial. A recolha de informação incluiu, ainda, entrevistas aos pais e consulta da documentação existente no processo da aluna, tendo esta consulta sido devidamente autorizado pelos pais da Bía e pela direção do Agrupamento de Escolas.

\section{A Bia}

Da história clínica e desenvolvimental da Bia salienta-se a gravidez normal, sempre vigiada. Contudo, no parto (ocorrido em 15/12/1997) verificou-se a necessidade de reanimação, tendo sido encaminhada para o Hospital Maria Pia do Porto, onde foi acompanhada em consultas de metabolismo e neuro-pediatria. Os exames neurológicos diagnosticaram Ataxia: perda de coordenação dos movimentos musculares voluntários; essa perda de coordenação pode ser causada por diversas condições médicas ou neurológicas, sendo a causa mais frequente a perda da função do cerebelo; a Ataxia pode comprometer os movimentos dos membros inferiores, dos membros superiores, do corpo, da fala ou dos olhos (NATIONAL ATAXIA FOUNDATION, 2010).

No caso da Bia, a Ataxia refletiu-se na falta de coordenação dos movimentos, afetando-lhe a força muscular e o equilíbrio, com alteração nas áreas desenvolvimentais, incidindo, principalmente, na produção da fala e na motricidade fina. A Bia não apresentava problemas de visão nem de audição. 
No ano letivo de 2009/2010, a Bia estava matriculada no 2. ${ }^{\circ}$ ano de escolaridade numa turma de 21 alunos e inserida na UAEM.

Em Setembro de 2008, os pais da Bia haviam aderido à iniciativa governamental de proporcionar um computador portátil (o computador Magalhães) a cada aluno designado por e-escolinha, mas o computador Magalhães chegou apenas em Maio de 2009, praticamente no final do ano letivo, razão pela qual, nesta fase do ano letivo, não foi concebida qualquer iniciativa para o computador ser usado na UAEM.

\section{Uso diário do computador Magalhães}

Em reunião com os pais da Bia, no início do ano letivo de 2009/10, estes referiram o seu novo interesse - o computador Magalhães - e, em função desta motivação, foi decidido delinear um programa de intervenção, potenciando os recursos disponíveis aos diferentes níveis, especialmente na área da comunicação. Foi assumido que as tecnologias poderiam vir a ser um facilitador de aprendizagens, atendendo às suas dificuldades motoras e aos seus limitados níveis de atenção e de concentração. Assim, acordou-se que a Bia transportaria todos os dias o computador para a escola, dado que o uso do computador fixo existente na UAEM estava limitado ao espaço de uma só sala.

A partir de outubro de 2009, a Bia explorou o computador Magalhães de forma sistemática treinando competências básicas: abrir o computador, ligá-lo, escolher o sistema operativo e copiar para o processador de texto palavras e frases simples. Todos os dias se sentava com o computador e, com muitas ajudas físicas e verbais ia, claramente, tendo mais facilidade na execução das pequenas tarefas que lhe eram sugeridas.

Em novembro de 2009, para um maior controle dos resultados obtidos e monitorização do processo em curso, foram concebidas duas Tabelas de Observação TIC (ver Anexo A) permitindo uma verificação da evolução das suas aquisições nesta área. Inicialmente, uma vez por semana, e, mensalmente, a partir do mês de janeiro de 2010, nessas tabelas eram registados os vários passos desde o "Abre computador" até ao "Aguarda o encerramento para o fechar", incluindo o reconhecimentos das letras do abecedário, dos números, dos sinais de acentuação e de pontuação.

\section{Avaliação de competências gerais de desenvolvimento, por um Centro de Recursos TIC (CRTIC)}

Em junho de 2009 e na sequência da aquisição do computador Magalhães, foi elaborado um pedido dirigido ao Centro de Recursos TIC (CRTIC) mais próximo para uma avaliação à Bia (os CRTIC são estruturas que têm, entre outros, como objetivo avaliar alunos com NEE de carácter permanente para fins de adequação das tecnologias de apoio às suas necessidades específicas). Uma das professoras responsáveis pediu à aluna para se sentar em frente ao computador e a avaliação das suas competências, ao nível das tecnologias, consistiu na observação do seu desempenho face à satisfação de uma série de indicações que lhe iam sendo dadas. 
Desta avaliação resultou que, mediante a problemática da Bia, deveria ser adquirido um computador portátil com um software adequado às suas necessidades, juntamente com o comunicador Go Talk 20+, que se considerou ser um meio para a aluna poder comunicar com os outros de forma eficaz e simples, por ser muito leve, resistente e concebido para transporte fácil. A Bia, seus pais e a UAEM ficaram a aguardar a receção do relatório para dar início à segunda fase do processo: a prescrição formal do equipamento e dos programas considerados adequados pela avaliação do CRTIC.

\section{Mudança de computador portátil}

Entretanto, durante a exploração do computador Magalhães, constatou-se que as limitações da Bia ao nível da realização de movimentos finos comprometiam a sua correta utilização, dada a dimensão reduzida do teclado. Foi assim considerado que a Bia necessitava de um computador com outras dimensões, em sequência foi formulado um pedido à Direção Regional de Educação Norte, elaborado ao abrigo do Despacho n. ${ }^{\circ}$ 2027/2010 de 29 de janeiro e fundamentado no Decreto-Lei n. ${ }^{\circ}$ 3/2008 de 7 de janeiro.

Na ausência de resposta, a Universidade do Minho colaborou nas diligências para a cedência de um computador portátil com um monitor de 15 polegadas. A intervenção começou a fazer-se com este computador e foram acrescentadas novas tarefas às Tabelas de Observação TIC.

O entusiasmo com que a Bia recebeu o novo computador foi patente na alegria com que descobriu certos pormenores, como aconteceu com "Os Jogos da Mimocas", quando conseguiu concluir o puzzle do corpo humano, pois já visualizava claramente os detalhes. Verificou-se, também, que era mais rápida a encontrar as letras no teclado e os sinais auxiliares de escrita e de acentuação. Por outro lado, conseguir abrir o computador demorou algum tempo e necessitou de muito treino e ajudas físicas. O facto de ser necessário pressionar um botão para abrir o computador e isto obrigar a um movimento coordenado das duas mãos, em que com o polegar de uma mão tinha que puxar o botão para a direita, ao mesmo tempo que a outra mão levantava o monitor, foi para a Bia uma tarefa complexa. No final da intervenção, apenas na escrita da password se verificavam algumas dificuldades. Numa qualquer atividade consistindo em copiar frases, acontecia premir demasiado uma determinada tecla e, consequentemente, escrever duas ou três letras seguidas; nesta situação, a Bia de imediato reformulava o que escrevia mas, no caso específico da password, ao premir excessivamente a tecla, como as letras não apareciam na janela do monitor (apenas visionava pontos), mesmo tendo noção do engano, não visualizava o erro.

Apesar da persistência de algumas dificuldades (como na situação da introdução da password) a Bia adquiriu, em tempo relativamente curto, dada a sua condição, a grande maioria dos procedimentos necessários para uma utilização quase autónoma do computador. 


\section{Introdução de sintetizadores de voz}

Verificando o envolvimento e a facilidade com que a Bia adquiria competências, ao nível das tecnologias, em outubro de 2009 foi proposto um produto de apoio de forma a promover e aumentar as suas competências comunicativas ao nível da expressão, através da introdução de um software com saída de voz com base em fala sintetizada (produzida por um dispositivo que converte informação matemática pré-programada acerca dos parâmetros do trato vocal, numa reprodução audível da voz humana). Contudo, após pesquisa e avaliação do software disponível no mercado, verificou-se que o seu elevado custo era uma barreira intransponível atendendo à situação económica dos pais da Bia. Entretanto, após uma pesquisa na Internet, surgiu um software de download grátis: o Sapis 15 TTSAPP, que imediatamente foi instalado no computador. Mas, para sintetizar as palavras, este software recorria a fonemas gravados português não europeu, o que resultava numa reprodução pouco inteligível e, em alguns casos, incorreta. Dois meses após a experimentação deste sintetizador e verificando-se que a Bia conseguia entender o seu funcionamento foi proposta a introdução de outro programa: o Linguatec Voice Reader 1.0.0.1 que, proporcionando uma boa qualidade ao nível da reprodução dos fonemas, passou, a partir desse momento, a ser utilizado pela criança nas atividades académicas.

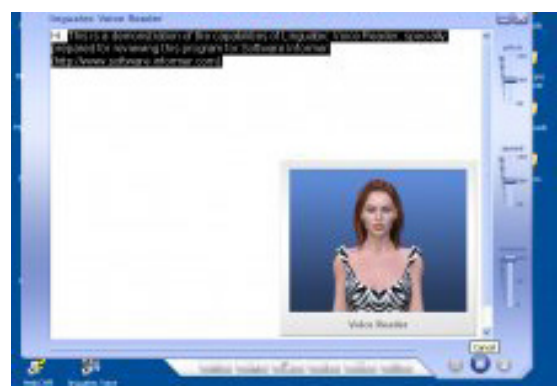

Figura 1 - Aspeto gráfico do programa Linguatec Voice Reader 1.0.0.1

Este sintetizador de voz (SV) possibilitava a ativação de um Avatar animado (optou-se por uma figura/voz do género feminino) e logo na primeira utilização desencadeou, na Bia, uma reação muito positiva e surpreendente: enquanto escrevia interagia com "ela", como se fosse uma personagem, se tratasse, cumprimentava-a, pedia-lhe para esperar e até se desculpava pelos lapsos.

As atividades desenvolvidas tiveram por base o seu perfil de funcionalidade, ou seja, toda a intervenção foi realizada em torno das suas competências individuais, com todas as adaptações necessárias para a sua execução. E, sendo a Bia uma utilizadora com problemas na motricidade fina e controle motor, configuraramse nas "Opções de Acessibilidade" e no "Painel de Controle", o tempo de repetição da tecla, o tempo de pressão e de libertar/premir repetidas vezes a mesma tecla. No "Controlador do Rato" também se modificou a velocidade e a aceleração do cursor, diferenciando entre a velocidade horizontal e vertical, o tempo de aceitação do clic e o tempo entre dois clics. 
A cópia para o SV tinha por objetivo auxiliar a Bia a compreender as possibilidades do código escrito. Todos os dias era providenciada uma rotina na qual as expetativas eram claras, consistentes e com a flexibilidade necessária para que a Bia conseguisse adaptar-se a mudanças que ocorriam com frequência e pudesse tomar decisões no processo. O trabalho desenvolvido, numa zona da sala mais isolada (área de trabalho individual), evitava estímulos distratores que a dispersassem e melhorava a sua capacidade de estar mais tempo em tarefa. Para aumentar a sua compreensão no que era pedido e para ilustração das palavras, tornando a atividade mais atrativa, recorreu-se a imagens desenhadas, fotografias, desenhos, etc. A forma de gerir e dinamizar esta atividade obedeceu, quase sempre, a uma sequência de etapas estruturadas: apresentação e identificação da imagem (imagem desenhada ou fotografia); identificação da palavra (escrita pela professora numa folha, em maiúsculas e com letra de imprensa); cópia da palavra para o SV e sua reprodução. O que a motivava era escrever sobre o que a rodeava e, quando se lhe perguntava sobre o que queria escrever, apontava para os colegas ou adultos em funções na UAEM. Às palavras soltas produzidas, seguiram-se frases muito simples e progressivamente mais complexas. Com a introdução da conjunção $[e]$ a Bia passou a produzir frases compostas e mais extensas que já incluíam vírgulas e acentuação.

\section{Prescrição de produtos de apoio}

No início de janeiro de 2010, já na posse do relatório do CRTIC, a direção do Agrupamento de Escolas, responsável por todos os procedimentos formais neste processo, contactou a Associação de Paralisia Cerebral (APC) da área, entidade prescritora aprovada, solicitando a prescrição do equipamento e programa escolhidos. A Coordenadora desta Instituição foi receptiva ao pedido, mas salientou que a aluna teria de ser avaliada pelos respectivos técnicos, em dia a agendar.

\section{Desenvolvimento do processo comunicacional com os pais}

Pelo fato de todos os dias o computador ser transportado entre a casa e a escola, foi criada uma pasta no ambiente de trabalho com a designação de Correio, que tinha como objetivo envolver ativamente os pais e estimular, na Bia, o gosto pela escrita, dando-lhe significado. A forma como isto se concretizou na prática foi muito simples: a Bia copiava, num ficheiro de texto, pequenos recados para os pais, com frases curtas e simples, sobre assuntos funcionais, do dia-a-dia. Os pais ou a professora inseriam tais frases no SV de forma a proporcionar uma audição e melhor compreensão das mensagens transmitidas. A responsabilidade da Bia, nesse processo, era alertar para a existência de recados e escrever, se necessário, a resposta.

Na intervenção ao nível das TIC, diversificaram-se os momentos e as estratégias, ao mesmo tempo que se procurou alternar as atividades de processamento de texto no SV, introduzindo outras atividades lúdicas. Embora a Bia só pudesse realizar alguns jogos, devido aos problemas motores, e nestes, apenas nos níveis mais baixos, essas atividades tinham como objetivo o desenvolvimento de diversas competências, como por exemplo, coordenação óculo-manual, a lateralidade, o treino da memória, da atenção e da concentração. 
Selecionou-se diversos programas educativos incluindo diferentes categorias de jogos e atividades: jogos de memória, puzzles, discriminação de cores, de agilidade, de pintura, de letras e de contagem. Começou-se por lhe proporcionar apenas um jogo, o Gcompris, e limitar a intervenção, evitando introduzir excesso de informação nova. Como referiu JOHNSON et al. (1999), citado por FARIA e CABRITA (2008, p. 179), a existência de um "agente pedagógico animado", com som, que fornecia um feedback positivo em resposta à ação do jogador era muito gratificante e motivador, funcionando como um reforço da aprendizagem. Mais tarde, instalou-se o Tux Paint, que foi utilizado pela Bia com relativa facilidade e, com o treino, foi variando de ferramentas e cores contrariando a tendência inicial de manter sempre a mesma.

O impacto que as tecnologias tiveram na Bia foi notório pelo seu empenho, pela capacidade de absorção de informação e autodeterminação. Este facto propiciou a expansão da ação, o que a conduziu ao acesso à Internet para fazer pesquisas de temas do seu interesse. Por exemplo, após a Bia escolher um animal, fazia-se a busca em "Imagens" que, depois de selecionadas, eram guardadas e legendadas numa pasta específica. Visitou, também, alguns sites de canções animadas e histórias digitais. Os colegas participaram nessas atividades que, muitas vezes, eram iniciadas em grupo para, posteriormente, prosseguirem apenas com a Bia.

Em Maio de 2009, a APC fez a prescrição solicitada sem a avaliação exigida inicialmente, após a Coordenadora ter decidido agilizar o processo, no sentido de corrigir toda uma série de mal entendidos prejudiciais à aluna e, tendo em conta a aproximação do final do ano letivo. Na posse de todos os elementos, a Adjunta do Diretor, deu andamento ao processo. Contudo, no final do ano letivo, ainda se aguardava a sua conclusão e consequente atribuição das ajudas técnicas.

\section{Aprendizagens realizadas e considerações finais}

Traduzindo-se numa igualdade de oportunidades, é essencial uma educação de qualidade que assegure a transmissão de atitudes, conhecimentos e competências que a sociedade, como um todo, encara como importantes para todas as crianças e jovens.

Com o surgimento das TIC e a sua difusão vertiginosa, as nossas relações sociais e as nossas práticas registaram transformações diárias consideráveis. Aos professores é solicitada a implementação de práticas eficazes junto dos alunos com NEE, que requer repensar uma intervenção planejada de forma colaborativa entre todos os intervenientes da equipa multidisciplinar tendo por base as características e necessidades dos alunos. As mudanças pedagógicas, que nos são pedidas perante a necessidade de gerar situações de aprendizagem mais eficazes, podem ser potenciadas e facilitadas pelas TIC.

Possibilitar aos alunos com NEE o acesso às TIC é determinante para a equidade e qualidade da ação educativa, perante o paradigma educacional emergente, que é, atualmente, a inclusão de alunos com NEE. 
Para a Bia, o computador foi um substituto eficaz do lápis e papel e as tecnologias como utensílio no processo ensino-aprendizagem foram bastante eficazes, na medida em que com programas com diferentes modos comunicacionais revelou compreender as tarefas de forma mais rápida e mantendo-se mais tempo em atividade. Por outro lado, provou ser um bom catalisador de motivação e uma ferramenta estimulante pelo seu dinamismo e interatividade, permitindo a intervenção a vários níveis.

Os diplomas legais desempenham um papel regulador indispensável à organização e funcionamento das atividades académicas, mas por si só não são suficientes enquanto existirem condicionalismos de ordem financeira, de ordem pessoal (atitudes) e de ordem profissional (falta de formação).

As mudanças qualitativas a promover devem ter por base variados fatores e processos de origem motivacional, cognitiva e afetiva, cuja interação é muito influente nas atitudes e nas decisões dos profissionais e pais.

\section{Referências}

CABRITA, I.; \& FARIA, J. Agentes pedagógicos animados em ambientes interactivos de aprendizagem. In: DIAS, P.; OSÓRIO, A., J. (Ed) Ambientes Educativos Emergentes. Universidade do Minho, Centro de Competências, 2008, p. 177-199.

CORREIA, L. Inclusão e necessidades educativas especiais: Um guia para educadores e professores. Porto: Porto Editora, 2008.

MINISTÉRIO DA EDUCAÇÃO. Decreto-Lei 3/2008, de 7 de Janeiro de 2008. Diário da República, N.․ 4, 1. ª série. Despacho n. 2027/2010, II Série, n. 20, de 29 de Janeiro. Diário da República, N. 20, 2. série. Instituto Nacional para a Reabilitação, I. P.

ILHARCO, F. A questão tecnológica, ensaio sobre a sociedade tecnológica contemporânea. PRINCIPIA, Publicações Universitárias e Cientificas, 2008.

NATIONAL ATAXIA FOUNDATION. Cognition and emotion in cerebellar disorders. Disponpivel em: 〈http://www.ataxia.org/〉, Acesso em: dez. 2010.

PAPERT, S., A. A máquina das crianças: repensando a escola na era da informática. Porto Alegre: Artes Médicas, 1994.

RODRIGUES, D. Paradigma da educação inclusiva: Reflexões sobre uma agenda possível. In Inclusão, l, 10-11. Braga: Universidade do Minho, 1997.

RODRIGUES, D.; MORATO, P.; \& MARTINS, R.; \& CLARA, H. As novas tecnologias na educação especial: Do assombro à realidade. In: IV Encontro Nacional de Educação Especial - Comunicações. Lisboa: Fundação Calouste Gulbenkian, 1991. 


\section{Anexos}

Tabelas a inserir Anexo A - Tabelas de Observação TIC

Tabela de Observação

\begin{tabular}{|c|c|c|c|c|c|c|c|c|c|c|c|c|c|}
\hline $\begin{array}{l}\text { Observação } \\
\text { feita em: }\end{array}$ & $09 / 10$ & $16 / 10$ & $23 / 10$ & $30 / 10$ & $12 / 11$ & $26 / 11$ & $11 / 12$ & $09 / 01$ & $23 / 01$ & $18 / 02$ & $20 / 03$ & $21 / 04$ & $19 / 05$ \\
\hline Abre a pasta & - & - & - & $\mathrm{NF}$ & F & $\mathrm{F}$ & F & F & $\mathrm{H}$ & $\mathrm{H}$ & $\mathrm{F}$ & F & $\mathrm{F}$ \\
\hline $\begin{array}{c}\text { Tira o } \\
\text { computador }\end{array}$ & - & - & - & NF & $\mathrm{NF}$ & $\mathrm{NF}$ & $\mathrm{NF}$ & NF & NF & $\mathrm{H}$ & $\mathrm{H}$ & F & F \\
\hline $\begin{array}{c}\text { Abre o } \\
\text { computador }\end{array}$ & F & F & F & $\mathrm{NF}$ & NF & $\mathrm{H}$ & F & F & F & F & F & F & F \\
\hline $\begin{array}{c}\text { Liga o } \\
\text { computador }\end{array}$ & F & $\mathrm{F}$ & F & $\mathrm{NF}$ & F & F & F & F & F & F & $\mathrm{F}$ & F & F \\
\hline $\begin{array}{l}\text { Aguarda pelas } \\
\text { definições }\end{array}$ & $\mathrm{H}$ & $\mathrm{H}$ & F & $\mathrm{H}$ & $\mathrm{H}$ & $\mathrm{F}$ & F & F & F & F & F & F & F \\
\hline $\begin{array}{l}\text { Escolhe } \\
\text { um sistema } \\
\text { operativo }\end{array}$ & $\mathrm{H}$ & $\mathrm{H}$ & F & & - & - & - & - & & - & - & - & - \\
\hline $\begin{array}{l}\text { Insere a pala- } \\
\text { vra-chave }\end{array}$ & - & - & & $\mathrm{NF}$ & $\mathrm{H}$ & $\mathrm{H}$ & $\mathrm{H}$ & $\mathrm{H}$ & $\mathrm{H}$ & F & $\mathrm{H}$ & $\mathrm{H}$ & F \\
\hline $\begin{array}{c}\text { Abre um } \\
\text { documento }\end{array}$ & $\mathrm{NF}$ & $\mathrm{NF}$ & $\mathrm{NF}$ & NF & $\mathrm{NF}$ & $\mathrm{NF}$ & $\mathrm{NF}$ & $\mathrm{NF}$ & $\mathrm{NF}$ & $\mathrm{NF}$ & $\mathrm{NF}$ & $\mathrm{NF}$ & $\mathrm{NF}$ \\
\hline $\begin{array}{c}\text { Copia } \\
\text { palavras }\end{array}$ & $\mathrm{H}$ & $\mathrm{H}$ & F & F & F & $\mathrm{F}$ & F & F & $\mathrm{F}$ & $\mathrm{F}$ & $\mathrm{F}$ & $\mathrm{F}$ & $\mathrm{F}$ \\
\hline $\begin{array}{c}\text { Copia frases } \\
\text { pequenas }\end{array}$ & $\mathrm{NF}$ & $\mathrm{NF}$ & $\mathrm{H}$ & $\mathrm{H}$ & F & $\mathrm{F}$ & F & F & $\mathrm{F}$ & $\mathrm{F}$ & $\mathrm{F}$ & $\mathrm{F}$ & $\mathrm{F}$ \\
\hline $\begin{array}{c}\text { Dá espaço } \\
\text { entre palavras }\end{array}$ & $\mathrm{NF}$ & $\mathrm{NF}$ & $\mathrm{H}$ & $\mathrm{H}$ & F & $\mathrm{F}$ & F & $\mathrm{F}$ & $\mathrm{F}$ & $\mathrm{F}$ & $\mathrm{F}$ & $\mathrm{F}$ & $\mathrm{F}$ \\
\hline $\begin{array}{l}\text { Muda de } \\
\text { linha (faz } \\
\text { parágrafo) }\end{array}$ & $\mathrm{NF}$ & $\mathrm{NF}$ & $\mathrm{H}$ & $\mathrm{F}$ & F & $\mathrm{F}$ & F & F & $\mathrm{F}$ & $\mathrm{F}$ & $\mathrm{F}$ & $\mathrm{F}$ & $\mathrm{F}$ \\
\hline $\begin{array}{c}\text { Fecha os } \\
\text { avisos } \\
\text { (antivírus,), os } \\
\text { documentos,... }\end{array}$ & F & F & F & F & F & F & F & F & F & F & $\mathrm{F}$ & F & F \\
\hline $\begin{array}{c}\text { Altera o tama- } \\
\text { nho da letra }\end{array}$ & $\mathrm{NF}$ & $\mathrm{NF}$ & $\mathrm{NF}$ & $\mathrm{H}$ & $\mathrm{H}$ & $\mathrm{H}$ & $\mathrm{H}$ & $\mathrm{H}$ & $\mathrm{H}$ & $\mathrm{H}$ & $\mathrm{H}$ & $\mathrm{H}$ & F \\
\hline $\begin{array}{c}\text { Altera a fonte } \\
\text { da letra }\end{array}$ & $\mathrm{NF}$ & $\mathrm{NF}$ & $\mathrm{NF}$ & NF & NF & $\mathrm{NF}$ & NF & $\mathrm{NF}$ & NF & $\mathrm{H}$ & $\mathrm{H}$ & $\mathrm{H}$ & $\mathrm{H}$ \\
\hline $\begin{array}{l}\text { Corrige } \\
\text { (apaga) os } \\
\text { erros }\end{array}$ & $\mathrm{NF}$ & & $\mathrm{H}$ & $\mathrm{F}$ & $\mathrm{F}$ & $\mathrm{F}$ & $\mathrm{F}$ & F & $\mathrm{F}$ & $\mathrm{F}$ & $\mathrm{F}$ & $\mathrm{F}$ & $\mathrm{F}$ \\
\hline $\begin{array}{c}\text { Põe o } \\
\text { computador a } \\
\text { carregar }\end{array}$ & $\mathrm{NF}$ & & NF & F & F & F & F & F & F & $\mathrm{F}$ & $\mathrm{F}$ & $\mathrm{F}$ & $\mathrm{F}$ \\
\hline $\begin{array}{c}\text { Guarda os } \\
\text { documentos }\end{array}$ & $\mathrm{NF}$ & & NF & $\mathrm{NF}$ & $\mathrm{H}$ & $\mathrm{H}$ & F & F & F & F & $\mathrm{F}$ & $\mathrm{F}$ & F \\
\hline $\begin{array}{c}\text { Desliga o } \\
\text { computador }\end{array}$ & $\mathrm{H}$ & & $\mathrm{H}$ & $\mathrm{F}$ & $\mathrm{F}$ & $\mathrm{F}$ & $\mathrm{F}$ & F & $\mathrm{F}$ & $\mathrm{F}$ & $\mathrm{F}$ & $\mathrm{F}$ & $\mathrm{F}$ \\
\hline $\begin{array}{c}\text { Aguarda o } \\
\text { encerramento } \\
\text { p/fechar }\end{array}$ & $\mathrm{NF}$ & $\mathrm{H}$ & $\mathrm{H}$ & $\mathrm{F}$ & F & $\mathrm{F}$ & $\mathrm{F}$ & $\mathrm{F}$ & $\mathrm{F}$ & $\mathrm{F}$ & $\mathrm{F}$ & $\mathrm{F}$ & $\mathrm{F}$ \\
\hline
\end{tabular}

Revista Educação Especial | v. 27 | n. 48 | p. 249-262 | jan./abr. 2014

\section{Santa Maria}


Legenda: F-Faz H-Hesita NF-Nãofaz

*30/10 - 30/10 - A Bia utiliza pela $1^{\underline{a}}$ vez um computador de 15 polegadas.

*23/01 - O saco onde o pc estava guardado não era apropriado por ser muito pequeno o que dificultava a tarefa de o tirar. A partir desta data passa a utilizar uma pasta com medidas adequadas.

Tabela de Observação TIC 2:

\begin{tabular}{|c|c|c|c|c|c|c|c|c|c|c|c|c|c|}
\hline \multicolumn{14}{|c|}{ Encontra as Letras } \\
\hline \multicolumn{14}{|c|}{ Observação feita em: } \\
\hline & $09 / 10$ & $16 / 10$ & $23 / 10$ & $30 / 10^{*}$ & $12 / 11$ & $26 / 11$ & $11 / 12$ & $09 / 01$ & $23 / 01^{*}$ & $18 / 02$ & $20 / 03$ & $21 / 04$ & $19 / 05$ \\
\hline A & $\mathrm{H}$ & F & F & F & F & F & F & F & F & F & F & F & F \\
\hline $\mathrm{B}$ & $\mathrm{H}$ & F & F & F & F & F & F & F & F & F & F & F & F \\
\hline $\mathrm{C}$ & & $\mathrm{H}$ & $\mathrm{H}$ & F & F & F & F & $\mathrm{F}$ & F & F & F & F & F \\
\hline $\mathrm{D}$ & NF & $\mathrm{H}$ & $\mathrm{H}$ & $\mathrm{H}$ & F & F & $\mathrm{H}$ & F & F & F & $\mathrm{H}$ & F & F \\
\hline $\mathrm{E}$ & $\mathrm{H}$ & $\mathrm{H}$ & F & F & F & F & F & F & F & $\mathrm{F}$ & F & F & F \\
\hline $\mathrm{F}$ & NF & & $\mathrm{H}$ & $\mathrm{H}$ & $\mathrm{H}$ & F & $\mathrm{H}$ & F & F & F & F & F & F \\
\hline G & NF & NF & $\mathrm{H}$ & F & $\mathrm{H}$ & F & $\mathrm{H}$ & F & F & $\mathrm{H}$ & F & $\mathrm{H}$ & $\mathrm{F}$ \\
\hline $\mathrm{H}$ & & & $\mathrm{H}$ & $\mathrm{H}$ & $\mathrm{F}$ & F & & & $\mathrm{H}$ & F & & $\mathrm{F}$ & $\mathrm{F}$ \\
\hline I & $\mathrm{H}$ & $\mathrm{F}$ & $\mathrm{F}$ & F & $\mathrm{F}$ & $\mathrm{F}$ & $\mathrm{F}$ & $\mathrm{F}$ & $\mathrm{F}$ & $\mathrm{F}$ & $\mathrm{F}$ & $\mathrm{F}$ & $\mathrm{F}$ \\
\hline $\mathrm{J}$ & & & & NF & $\mathrm{H}$ & $\mathrm{H}$ & $\mathrm{H}$ & & $\mathrm{H}$ & $\mathrm{H}$ & F & F & F \\
\hline $\mathrm{K}$ & & & & & & & & & & & NF & & \\
\hline $\mathrm{L}$ & & NF & $\mathrm{H}$ & $\mathrm{F}$ & & $\mathrm{F}$ & F & $\mathrm{F}$ & $\mathrm{F}$ & $\mathrm{F}$ & $\mathrm{F}$ & $\mathrm{F}$ & $\mathrm{F}$ \\
\hline M & $\mathrm{H}$ & F & F & F & F & F & $\mathrm{F}$ & F & F & F & F & F & F \\
\hline $\mathrm{N}$ & $\mathrm{H}$ & $\mathrm{H}$ & $\mathrm{F}$ & $\mathrm{F}$ & $\mathrm{H}$ & $\mathrm{F}$ & F & $\mathrm{F}$ & $\mathrm{F}$ & $\mathrm{F}$ & $\mathrm{F}$ & $\mathrm{F}$ & $\mathrm{F}$ \\
\hline $\mathrm{O}$ & $\mathrm{H}$ & F & F & F & $\mathrm{F}$ & F & F & F & $\mathrm{F}$ & F & F & F & F \\
\hline $\mathrm{P}$ & & NF & $\mathrm{H}$ & $\mathrm{F}$ & $\mathrm{F}$ & $\mathrm{F}$ & & $\mathrm{F}$ & $\mathrm{F}$ & F & F & F & F \\
\hline $\mathrm{Q}$ & & & & & NF & $\mathrm{H}$ & $\mathrm{H}$ & & $\mathrm{H}$ & F & F & & \\
\hline $\mathrm{R}$ & & $\mathrm{H}$ & $\mathrm{F}$ & F & $\mathrm{H}$ & $\mathrm{F}$ & F & F & $\mathrm{F}$ & F & F & F & F \\
\hline S & & NF & $\mathrm{H}$ & F & F & F & & F & F & F & F & F & F \\
\hline $\mathrm{T}$ & $\mathrm{H}$ & & $\mathrm{F}$ & $\mathrm{F}$ & F & F & & $\mathrm{F}$ & $\mathrm{F}$ & F & F & F & F \\
\hline $\mathrm{U}$ & & & NF & $\mathrm{H}$ & $\mathrm{H}$ & $\mathrm{F}$ & $\mathrm{F}$ & F & $\mathrm{H}$ & F & & $\mathrm{H}$ & \\
\hline V & & & & NF & NF & $\mathrm{H}$ & $\mathrm{H}$ & $\mathrm{H}$ & F & & $\mathrm{H}$ & & $\mathrm{H}$ \\
\hline \multicolumn{14}{|l|}{ W } \\
\hline$X$ & & & NF & $\mathrm{H}$ & F & F & & & F & & $\mathrm{H}$ & & \\
\hline \multicolumn{14}{|l|}{$\mathrm{Y}$} \\
\hline Z & & & & & & $\mathrm{H}$ & $\mathrm{H}$ & $\mathrm{F}$ & $\mathrm{F}$ & & F & & \\
\hline Ç & & NF & $\mathrm{NF}$ & & $\mathrm{H}$ & & & $\mathrm{H}$ & $\mathrm{H}$ & F & & & \\
\hline
\end{tabular}

Legenda: F- Faz H-Hesita NF-Nãofaz

*30/10 - 30/10 - A Bia utiliza pela 1ª vez um computador de 15 polegadas.

*23/01 - O saco onde o pc estava guardado não era apropriado por ser muito pequeno o que dificultava a tarefa de tirar. A partir desta data passa a utilizar uma pasta com medidas adequadas. 
Tabela de Observação TIC 3:

\begin{tabular}{|c|c|c|c|c|c|c|c|c|c|c|c|c|}
\hline \multicolumn{10}{|c|}{ Encontra os sinais de pontuação } \\
\hline \multicolumn{10}{|c|}{ Observação feita em: } \\
\hline & $09 / 10$ & $16 / 10$ & $23 / 10$ & $30 / 10^{*}$ & $12 / 11$ & $26 / 11$ & $11 / 12$ & $09 / 01$ & $23 / 01^{*}$ & $18 / 02$ & $20 / 03$ & $19 / 05$ \\
\hline & NF & NF & NF & NF & NF & NF & H & H & H & F & F & F \\
\hline ^ & NF & NF & & NF & & NF & & & H & H & & H \\
\hline$\cdot$ & NF & NF & H & H & H & H & F & F & F & F & F & F \\
\hline- & NF & NF & NF & NF & H & H & & H & H & H & & \\
\hline$?$ & & & NF & NF & & NF & NF & H & H & & H & \\
\hline$!$ & NF & & & NF & NF & & & H & H & & H & \\
\hline$:$ & NF & NF & & NF & H & & H & H & F & & H & \\
\hline, & & NF & NF & NF & NF & NF & H & H & F & H & H & \\
\hline
\end{tabular}

Legenda: F- Faz H - Hesita NF- Nãofaz

*30/10 - 30/10 - A Bia utiliza pela $1^{\text {a }}$ vez um computador de 15 polegadas.

*23/01 - O saco onde o pc estava guardado não era apropriado por ser muito pequeno o que dificultava a tarefa de o tirar. A partir desta data passa a utilizar uma pasta com medidas adequadas.

Tabela de Observação TIC 4:

\begin{tabular}{|c|c|c|c|c|c|c|c|c|c|c|c|c|}
\hline \multicolumn{10}{|c|}{ Encontra os números } \\
\hline \multicolumn{10}{|c|}{ Observação feita em: } \\
\hline & $09 / 10$ & $16 / 10$ & $23 / 10$ & $30 / 10$ & $12 / 11$ & $26 / 11$ & $11 / 12$ & $09 / 01$ & $23 / 01$ & $18 / 02$ & $20 / 03$ & $19 / 05$ \\
\hline 1 & H & F & F & F & F & F & F & F & F & F & F & F \\
\hline 2 & H & F & F & F & F & F & F & F & F & F & F & F \\
\hline 3 & H & F & F & F & F & F & F & F & F & F & F & F \\
\hline 4 & H & H & F & F & F & F & H & F & F & F & F & F \\
\hline 5 & H & F & F & F & F & F & F & F & F & F & F & F \\
\hline 6 & H & H & F & F & F & F & F & F & F & F & F & F \\
\hline 7 & H & F & F & F & H & F & H & F & F & F & F & F \\
\hline 8 & F & F & F & F & F & F & H & F & F & F & F & F \\
\hline 9 & H & H & F & H & F & F & F & F & H & F & F & H \\
\hline 0 & H & F & F & F & F & F & H & F & F & F & F & F \\
\hline
\end{tabular}

Legenda: F-Faz H-Hesita NF-Nãofaz

*30/10 - 30/10 - A Bia utiliza pela 1ª vez um computador de 15 polegadas.

*23/01 - O saco onde o pc estava guardado não era apropriado por ser muito pequeno o que dificultava a tarefa de o tirar. A partir desta data passa a utilizar uma pasta com medidas adequadas. 